\title{
Bivariate odd-log-logistic-Weibull regression model for oral health-related quality of life
}

\author{
José N. da Cruz ${ }^{a}$, Edwin M. M. Ortega ${ }^{a}$, Gauss M. Cordeiro ${ }^{b}$, Adriano K. Suzuki ${ }^{1, c}$, \\ Fábio L. Mialhe ${ }^{d}$ \\ ${ }^{a}$ Department of Exact Sciences, ESALQ-USP, Brazil; ${ }^{b}$ Department of Statistics, UFPE, Brazil; \\ ${ }^{c}$ Department of Applied Mathematics and Statistics, ICMC-USP, Brazil; \\ ${ }^{d}$ Department of Community Dentistry, Division of Health Education and Health Promotion, \\ UNICAMP, Brazil
}

\begin{abstract}
We study a bivariate response regression model with arbitrary marginal distributions and joint distributions using Frank and Clayton's families of copulas. The proposed model is used for fitting dependent bivariate data with explanatory variables using the log-odd log-logistic Weibull distribution. We consider likelihood inferential procedures based on constrained parameters. For different parameter settings and sample sizes, various simulation studies are performed and compared to the performance of the bivariate odd-log-logistic-Weibull regression model. Sensitivity analysis methods (such as local and total influence) are investigated under three perturbation schemes. The methodology is illustrated in a study to assess changes on schoolchildren's oral health-related quality of life (OHRQoL) in a follow-up exam after three years and to evaluate the impact of caries incidence on the OHRQoL of adolescents.
\end{abstract}

Keywords: Clayton copula, Frank copula, local influence, regression model, Weibull distribution

\section{Introduction}

In statistical analysis, bivariate longitudinal data may represent the occurrence of successive events within the same individual. There is a probably dependence between these events and its investigation may be the main interest in a medical trial. Individual models for each event are based on independence assumptions and do not allow for inferences in a possible association. The use of bivariate models seem more adequate and this approach has being used under different approaches and can be found in Barriga et al. (2010), Chatterjee and Shih (2001), Fachini et al. (2014), and Núñez (2005). Besides the use of the classical multivariate parametric distributions, copulas can also be used to join marginal models into multivariate models. Flexibility is provided by the copulas on the marginal selection results in uncountable distributions with distinct properties. In this paper, Frank and Clayton's families of copulas are used to construct linear location-scale marginal models including covariates in the modeling of survival data. For marginal distributions, we consider the log-odd-log-logisticWeibull (LOLLW) distribution, which is a recent generalization of the Weibull distribution (da Cruz et al., 2016).

\footnotetext{
${ }^{1}$ Corresponding author: Department of Applied Mathematics and Statistics, University of São Paulo, Av. Trabalhador São-carlense, 400 - Centro CEP: 13566-590, São Carlos, SP, Brazil. E-mail: suzuki@icmc.usp.br
}

Published 31 May 2017 / journal homepage: http://csam.or.kr

(c) 2017 The Korean Statistical Society, and Korean International Statistical Society. All rights reserved. 
The inferential part of the work is conducted using the maximum likelihood theory and the asymptotic properties of the estimators. Influence diagnostics represents is an important step in the analysis of a dataset since they provide an indication of lack-of-fit model or of influential observations. However, there are no applications of influence diagnostic procedures to the bivariate regression model. Cook (1986) introduced a diagnostic approach called local influence to assess the effects of small perturbations in the model and/or data on the parameter estimates. Several authors have applied the local influence method in more general regression models than the normal regression model. Some others have also explored the assessment of local influence in survival analysis models. For instance, Pettitt and Bin Daud (1989) investigated local influence in proportional hazard regression models, Escobar and Meeker (1992) adapted local influence methods to regression analysis under censoring, Silva et al. (2010) considered the problem of assessing local influence in log-Weibull (LW) extended regression model, Hashimoto et al. (2013) investigated global and local influence in log-generalized gamma regression model for interval-censored data and Ortega et al. (2013) applied local influence in the log-beta Weibull regression model with application to predict the recurrence of prostate cancer. Recently, Ortega et al. (2015) adapted local influence methods to a power series beta Weibull regression model to predict breast carcinoma, Hashimoto et al. (2015) studied local influence in a long-term survival model with interval-censored data and Ortega et al. (2017) applied local influence in regression models generated by gamma random variables. We propose a similar method to detect influential subjects by considering the local influence approach for the bivariate LOLLW regression model.

The paper is organized as follows. In Section 2, we discuss bivariate response models using Frank and Clayton's families and the LOLLW distribution. In Section 3, we propose the Frank-LOLLW and Clayton-LOLLW bivariate regression models and the inference strategy based on maximum likelihood estimates (MLEs) and present a simulation study. In Section 4, we study some diagnostic measures by considering case deletion and the normal curvatures of local influence, and derive the likelihood function under different perturbation schemes for the bivariate response regression models. In Section 5 , the proposed methods are applied to a real dataset. Section 6 presents the concluding remarks.

\section{Bivariate response models using Frank-LOLLW and Clayton-LOLLW copulas}

We consider the models introduced by He and Lawless (2005) that have bivariate response variables with joint distribution given by

$$
F\left(y_{1}, y_{2}\right)=C_{\lambda}\left(\frac{y_{1}-\mu_{1}}{\sigma_{1}}, \frac{y_{2}-\mu 2}{\sigma_{2}}\right),
$$

where $C$ is a copula function on $\mathbb{R}^{2}, \lambda$ is the association parameter and the marginal distributions of $Y_{1}$ and $Y_{2}$ have the location-scale form

$$
Y_{k}=\mu_{k}+z_{k}
$$

where $\mu_{1} \in \mathbb{R}$ and $\mu_{2} \in \mathbb{R}$ are location parameters, $\sigma_{1}>0$ and $\sigma_{2}>0$ are the scale parameters and $z_{1}$ and $z_{2}$ are the model errors.

Copulas are functions that provide means to create multivariate distribution functions with different dependence structures based on arbitrary marginal functions. Let $Y_{k}$ be a random variable with a continuous marginal distribution $F_{k}$ for $k=1,2$. The joint distribution of $\left(Y_{1}, Y_{2}\right)$ is given by

$$
F\left(y_{1}, y_{2}\right)=C_{\lambda}\left\{F_{1}\left(y_{1}\right), F_{2}\left(y_{2}\right)\right\}
$$


conditioned on $F_{k}\left(y_{k}\right) \sim U(0,1), \forall k$. The joint distribution $C_{\lambda}$ describes the dependence of the random variables $Y_{1}$ and $Y_{2}$ through the association parameter $\lambda$.

Various copula functions could have been used in this study. However, the most often cited are the Frank (1979) and Clayton (1978) copulas for $k=2$. Of course many other copulas exist and details can be found in other texts such as Nelsen (2006). The expressions for these selected copulas are presented with some limit properties used to create bivariate response regression models. This class of copula is widely used and has many attractive properties. For more details, see Genest (1987) and Nelsen (2006).

- Frank family

The joint distribution function for the Frank family is given by

$$
C_{\lambda}\left(y_{1}, y_{2}\right)=-\frac{1}{\lambda} \log \left\{1+\frac{\left\{\exp \left[-\lambda F_{1}\left(y_{1}\right)\right]-1\right\}\left\{\exp \left[-\lambda F_{2}\left(y_{2}\right)\right]-1\right\}}{\exp (-\lambda)-1}\right\},
$$

where $\lambda \in \mathbb{R} \backslash\{0\}$. For $\lambda \rightarrow 0$, we obtain $C_{0}\left(y_{1}, y_{2}\right)=F_{1}\left(y_{1}\right) F_{2}\left(y_{2}\right)$ with arbitrary marginal functions $F_{1}\left(y_{1}\right)$ and $F_{2}\left(y_{2}\right)$. For $\lambda \rightarrow-\infty$, we obtain $C_{-\infty}\left(y_{1}, y_{2}\right)=\max \left(F_{1}\left(y_{1}\right)+F_{2}\left(y_{2}\right)-1,0\right)$ and, similarly, for $\lambda \rightarrow \infty$, we obtain $C_{\infty}\left(y_{1}, y_{2}\right)=\min \left(F_{1}\left(y_{1}\right), F_{2}\left(y_{2}\right)\right)$;

- Clayton family

The joint distribution function for the Clayton family is given by

$$
C_{\lambda}\left(y_{1}, y_{2}\right)=\left\{F_{1}\left(y_{1}\right)^{-\lambda}+F_{2}\left(y_{2}\right)^{-\lambda}-1\right\}^{-\frac{1}{\lambda}},
$$

where $\lambda \in[-1,+\infty)$ and $\lambda \neq 0$. For $\lambda \rightarrow 0$, we obtain $C_{0}\left(y_{1}, y_{2}\right)=F_{1}\left(y_{1}\right) F_{2}\left(y_{2}\right)$. For $\lambda=1$, we have $C_{1}\left(y_{1}, y_{2}\right)=\max \left(F_{1}\left(y_{1}\right)+F_{2}\left(y_{2}\right)-1,0\right)$ and, similarly, for $\lambda \rightarrow \infty$, we obtain $C_{\infty}\left(y_{1}, y_{2}\right)=$ $\min \left(F_{1}\left(y_{1}\right), F_{2}\left(y_{2}\right)\right)$.

As can be seen in equations (2.3) and (2.4), we have to define the marginal distributions $F_{1}\left(y_{1}\right)$ and $F_{2}\left(y_{2}\right)$. In this paper, we adopt the LOLLW distribution described by Da Cruz et al. (2016).

In statistics, the Weibull and extreme value (LW) distributions are the most popular models for applications to real data. When the number of observations is large, they can be adopted as approximate distributions for other models. The probability density function (pdf) and cumulative distribution function (cdf) of the LW (for $y \in \mathbb{R}$ ) model are given by

$$
g(y ; \mu, \sigma)=\frac{1}{\sigma} \exp \left[\left(\frac{y-\mu}{\sigma}\right)-\exp \left(\frac{y-\mu}{\sigma}\right)\right] \quad \text { and } \quad G(y ; \mu, \sigma)=1-\exp \left[-\exp \left(\frac{y-\mu}{\sigma}\right)\right],
$$

where $\mu \in \mathbb{R}$ is a location parameter and $\sigma>0$ is a scale parameter.

The cdf of the LOLLW distribution with an additional shape parameter $\alpha>0$ is defined by

$$
F(y ; \mu, \sigma, \alpha)=\int_{0}^{\frac{G(y, \mu, \sigma)}{G(y ; \mu, \sigma)}} \frac{\alpha y^{\alpha-1}}{\left(1+y^{\alpha}\right)^{2}} d y=\frac{G(y ; \mu, \sigma)^{\alpha}}{G(y ; \mu, \sigma)^{\alpha}+\bar{G}(y ; \mu, \sigma)^{\alpha}},
$$

where $\bar{G}(y ; \mu, \sigma)=1-G(y ; \mu, \sigma)$.

The LW cdf $G(y ; \mu, \sigma)$ is clearly a special case of (2.6) when $\alpha=1$. We note that there is no complicated function in equation (2.6) in contrast with the beta generalized family (Eugene et al., 
2002), which includes two extra parameters and the incomplete beta function. The LOLLW density is given by

$$
f(y ; \mu, \sigma, \alpha)=\frac{\alpha g(y ; \mu, \sigma)\{G(y ; \mu, \sigma)[1-G(y ; \mu, \sigma)]\}^{\alpha-1}}{\left\{G(y ; \mu, \sigma)^{\alpha}+[1-G(y ; \mu, \sigma)]^{\alpha}\right\}^{2}} .
$$

We can write by omitting the parameters of the cdf

$$
\alpha=\frac{\log [F(y) / \bar{F}(y)]}{\log [G(y) / \bar{G}(y)]} \quad \text { and } \quad \bar{G}(y)=1-G(y) .
$$

Thus, the parameter $\alpha$ represents the quotient of the log odds ratio for the generated and baseline distributions. The LOLL-G family has received increased attention over the last few years, for example, Cordeiro et al. (2017a) considered odd log-logistic generalized half-normal lifetime distribution, Da Silva Braga et al. (2016) proposed odd log-logistic normal distribution, Ortega et al. (2016) developed the odd Birnbaum-Saunders regression model and recently Cordeiro et al. (2017b) presented the generalized odd log-logistic family of distributions.

The cdf and pdf of the LOLLW distribution can be expressed as

$$
F(y ; \mu, \sigma, \alpha)=\frac{\left\{1-\exp \left[-\exp \left(\frac{y-\mu}{\sigma}\right)\right]\right\}^{\alpha}}{\left\{1-\exp \left[-\exp \left(\frac{y-\mu}{\sigma}\right)\right]\right\}^{\alpha}+\left\{\exp \left[-\exp \left(\frac{y-\mu}{\sigma}\right)\right]\right\}^{\alpha}}
$$

and

$$
f(y ; \mu, \sigma, \alpha)=\frac{\alpha \exp \left(\frac{y-\mu}{\sigma}\right)\left\{\exp \left[-\exp \left(\frac{y-\mu}{\sigma}\right)\right]\right\}^{\alpha}\left\{1-\exp \left[-\exp \left(\frac{y-\mu}{\sigma}\right)\right]\right\}^{\alpha-1}}{\sigma\left\{\left\{1-\exp \left[-\exp \left(\frac{y-\mu}{\sigma}\right)\right]\right\}^{\alpha}+\left\{\exp \left[-\exp \left(\frac{y-\mu}{\sigma}\right)\right]\right\}^{\alpha}\right\}^{2}},
$$

respectively. Note that $\alpha>0$ is a shape parameter. Henceforth, a random variable with density function (2.9) is denoted by $Y \sim \operatorname{LOLLW}(\alpha, \mu, \sigma)$. For $\sigma=1$, we obtain the $\log$-odd $\log$-logistic exponential (LOLLE) distribution. Further, the LOLLE distribution with $\alpha=1$ reduces to the LW distribution.

The quantile function (qf) is widespread use in general statistics. Equation (2.6) has tractable properties specially for simulations, since its qf has a simple form

$$
y=Q_{Z}(v)=Q_{L W}\left(\frac{v^{\frac{1}{\alpha}}}{[1-v]^{\frac{1}{\alpha}}+v^{\frac{1}{\alpha}}}\right),
$$

where $z=Q_{L W}(v)=\mu+\sigma \log [-\log (1-v)]$ is the $\mathrm{LW}$ qf derived by inverting (2.5), i.e., $G(y ; \mu, \sigma)=v$.

If the marginal distributions of the model errors in (2.2) follow the LOLLW distribution and substituting (2.8) and (2.9) in equations (2.3) and (2.4), the joint cdfs are given by:

- Frank-LOLLW joint distribution function

$$
F_{F}\left(y_{1}, y_{2}\right)=-\frac{1}{\lambda} \log \left\{1+\frac{\left\{\exp \left[\frac{-\lambda\left(1-u_{1}\right)^{\alpha_{1}}}{\left(1-u_{1}\right)^{\alpha_{1}}+u_{1}^{\alpha_{1}}}\right]-1\right\}\left\{\exp \left[\frac{-\lambda\left(1-u_{2}\right)^{\alpha_{2}}}{\left(1-u_{2}\right)^{\alpha_{1}}+u_{2}^{\alpha_{2}}}\right]-1\right\}}{\exp (-\lambda)-1}\right\} .
$$




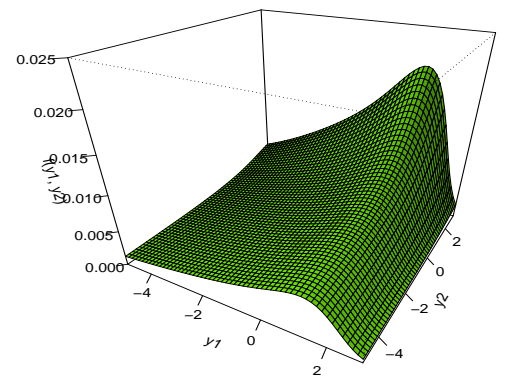

(a) $\lambda=0.01, \alpha_{1}=0.5, \alpha_{2}=0.5, \mu_{1}=0, \mu_{2}=0$, $\sigma_{1}=1.25, \sigma_{2}=2$

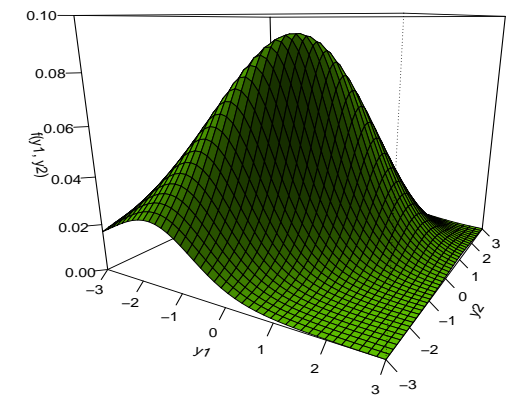

(b) $\lambda=5, \alpha_{1}=1.5, \alpha_{2}=1.3, \mu_{1}=0, \mu_{2}=0$, $\sigma_{1}=2, \sigma_{2}=2$

Figure 1: Probability density function of Frank's copula.

- Clayton-LOLLW joint distribution function

$$
F_{C}\left(y_{1}, y_{2}\right)=\left\{\left[\frac{\left(1-u_{1}\right)^{\alpha_{1}}}{\left(1-u_{1}\right)^{\alpha_{1}}+u_{1}^{\alpha_{1}}}\right]^{-\lambda}+\left[\frac{\left(1-u_{2}\right)^{\alpha_{2}}}{\left(1-u_{2}\right)^{\alpha_{2}}+u_{2}^{\alpha_{2}}}\right]^{-\lambda}-1\right\}^{-\frac{1}{\lambda}}
$$

The joint pdfs are given by:

- Frank-LOLLW joint density function

$$
\begin{aligned}
f_{F}\left(y_{1}, y_{2}\right)= & \frac{\alpha_{1} \alpha_{2} \exp \left(z_{1}+z_{2}\right) \lambda u_{1}^{\alpha_{1}} u_{2}^{\alpha_{2}}[-1+\exp (\lambda)] \exp \left\{\lambda\left[1+q_{1}+q_{2}\right]\right\}}{\left\{\exp (\lambda)-\exp \left[\lambda\left(1+q_{1}\right)\right]+\exp \left[\lambda\left(q_{1}+q_{2}\right)\right]-\exp \left[\lambda\left(1+q_{2}\right)\right]\right\}^{2}} \\
& \times \frac{\left(1-u_{1}\right)^{\alpha_{1}-1}\left(1-u_{2}\right)^{\alpha_{2}-1}}{\sigma_{1} \sigma_{2}\left[\left(1-u_{1}\right)^{\alpha_{1}}+u_{1}^{\alpha_{1}}\right]^{2}\left[\left(1-u_{2}\right)^{\alpha_{2}}+u_{2}^{\alpha_{2}}\right]^{2}}
\end{aligned}
$$

- Clayton-LOLLW joint density function

$$
f_{C}\left(y_{1}, y_{2}\right)=\frac{\alpha_{1} \alpha_{2} \exp \left(z_{1}+z_{2}\right) u_{1}^{\alpha_{1}} u_{2}^{\alpha_{2}}\left(1-u_{1}\right)^{\alpha_{1}}\left(1-u_{2}\right)^{\alpha_{2}}(1+\lambda)\left(q_{1} q_{2}\right)^{-(1+\lambda)}}{\sigma_{1} \sigma_{2}\left[\left(1-u_{1}\right)^{\alpha_{1}}+u_{1}^{\alpha_{1}}\right]^{2}\left[\left(1-u_{2}\right)^{\alpha_{2}}+u_{2}^{\alpha_{2}}\right]^{2}\left[q_{1}^{-\lambda}+q_{2}^{-\lambda}-1\right]^{\left(2+\frac{1}{\lambda}\right)}},
$$

where

$$
q_{k}=\frac{\left(1-u_{k}\right)^{\alpha_{k}}}{\left(1-u_{k}\right)^{\alpha_{k}}+u_{k}^{\alpha_{k}}}, \quad u_{k}=\exp \left[-\exp \left(z_{k}\right)\right], \quad z_{k}=\frac{y_{k}-\mu_{k}}{\sigma_{k}}, \quad \text { for } k=1,2 .
$$

Equations (2.13) and (2.14) are referred to as the Frank-LOLLW and Clayton-LOLLW bivariate models, respectively. The Frank-LOLLW and Clayton-LOLLW models contain as special cases several well-known distributions. For example, they simplify to the Frank-log-Weibull (Frank-LW) and Clayton-log-Weibull (Clayton-LW) bivariate models when $\alpha_{1}=\alpha_{2}=1$. If $\sigma_{1}=\sigma_{2}=1$, they reduce to the Frank-log odd log-logistic exponential (Frank-LOLLE) and Clayton-log odd loglogistic exponential (Clayton-LOLLE) bivariate models. If $\alpha_{1}=\alpha_{2}=1$, in addition to $\sigma_{1}=\sigma_{2}=1$, the Frank-LOLLW and Clayton-LOLLW models reduce to the Frank-log-exponential (Frank-LE) and Clayton-log-exponential (Clayton-LE) bivariate models. Plots of the pdf of the Frank's copula are displayed in Figure 1 and plots of the pdf of the Clayton's copula are displayed in Figure 2. 


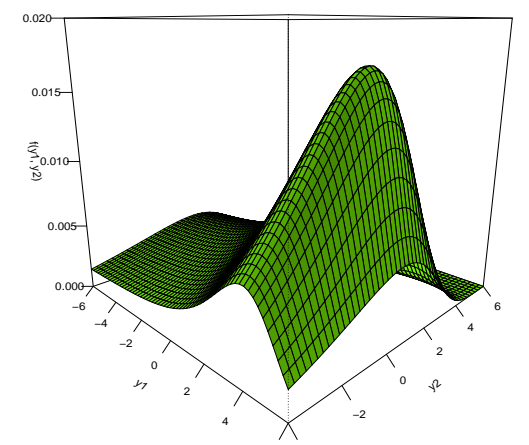

(a) $\lambda=0.1, \alpha_{1}=0.2, \alpha_{2}=0.75, \mu_{1}=0, \mu_{2}=0$, $\sigma_{1}=2, \sigma_{2}=2$

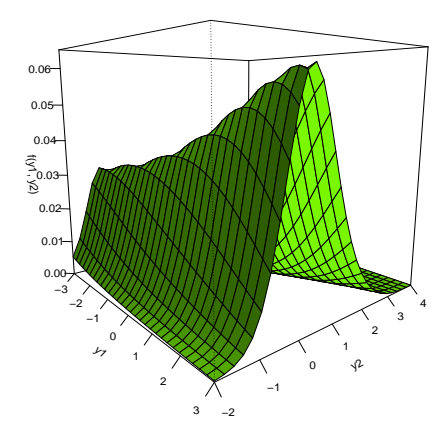

(b) $\lambda=5, \alpha_{1}=0.3, \alpha_{2}=1.5, \mu_{1}=0, \mu_{2}=0$, $\sigma_{1}=2, \sigma_{2}=2$

Figure 2: Probability density function of Clayton's copula.

\section{Regression model and inference strategies}

Let $Y_{1}$ and $Y_{2}$ be two random variables related to a bivariate response of an individual and consider that there is a linear relation between the variables $Y_{k}(k=1,2)$ and a vector of explanatory variables $\boldsymbol{x}_{k}=\left(x_{1 k}, \ldots, x_{p k}\right)^{T}$. A log-linear regression model can be defined by

$$
Y_{k}=\boldsymbol{x}_{k}^{T} \boldsymbol{\beta}_{k}+\sigma_{k} z_{k},
$$

where $\beta_{1}$ and $\beta_{2}$ are the regression coefficients associated with the vectors of explanatory variables $\boldsymbol{x}_{1}$ and $\boldsymbol{x}_{2}$ with dimension $p, \sigma_{1}>0$ and $\sigma_{2}>0$ are the scale parameters and $z_{1}$ and $z_{2}$ are the model errors with joint distribution given by equations (2.13) and (2.14) and independent of $\boldsymbol{x}_{1}$ and $\boldsymbol{x}_{2}$. If $z_{1}$ and $z_{2}$ are independent, the model given in (2.2) is reduced to a traditional location-scale regression model.

Consider a sample $\left(y_{1 k}, \boldsymbol{x}_{1 k}\right), \ldots,\left(y_{n k}, \boldsymbol{x}_{n k}\right)$ (for $\left.k=1,2\right)$ of $n$ independent observations, the model given in (3.1) and the joint pdfs given in equations (2.13) and (2.14). The total log-likelihood function for the parameter $\boldsymbol{\psi}=\left(\lambda, \boldsymbol{\theta}_{1}^{T}, \boldsymbol{\theta}_{2}^{T}\right)^{T}$, where $\boldsymbol{\theta}_{k}=\left(\boldsymbol{\beta}_{k}^{T}, \sigma_{k}, \alpha_{k}\right)^{T}$ and $\boldsymbol{\beta}_{k}^{T}=\left(\beta_{1 k}, \ldots, \beta_{p k}\right)($ for $k=1,2)$, is given by:

- Frank-LOLLW model

$$
\begin{aligned}
l(\boldsymbol{\psi})= & n \log \left\{\frac{\lambda \alpha_{1} \alpha_{2}[\exp (\lambda)-1]}{\sigma_{1} \sigma_{2}}\right\}+\sum_{i=1}^{n}\left(z_{i 1}+z_{i 2}\right)+\alpha_{1} \sum_{i=1}^{n} \log \left(u_{i 1}\right)+\alpha_{2} \sum_{i=1}^{n} \log \left(u_{i 2}\right) \\
& +\lambda \sum_{i=1}^{n}\left(1+q_{i 1}+q_{i 2}\right)+\left(\alpha_{1}-1\right) \sum_{i=1}^{n} \log \left(1-u_{i 1}\right)+\left(\alpha_{2}-1\right) \sum_{i=1}^{n} \log \left(1-u_{i 2}\right) \\
& -2 \sum_{i=1}^{n} \log \left\{\exp (\lambda)-\exp \left[\lambda\left(1+q_{i 1}\right)\right]+\exp \left[\lambda\left(q_{i 1}+q_{i 2}\right)\right]-\exp \left[\lambda\left(1+q_{i 2}\right)\right]\right\} \\
& -2 \sum_{i=1}^{n} \log \left[\left(1-u_{i 1}\right)^{\alpha_{1}}+u_{i 1}^{\alpha_{1}}\right]-2 \sum_{i=1}^{n} \log \left[\left(1-u_{i 2}\right)^{\alpha_{2}}+u_{i 2}^{\alpha_{2}}\right] ;
\end{aligned}
$$


- Clayton-LOLLW model

$$
\begin{aligned}
l(\boldsymbol{\psi})= & n \log \left[\frac{(1+\lambda) \alpha_{1} \alpha_{2}}{\sigma_{1} \sigma_{2}}\right]+\sum_{i=1}^{n}\left(z_{i 1}+z_{i 2}\right)+\alpha_{1} \sum_{i=1}^{n} \log \left(u_{i 1}\right)+\alpha_{2} \sum_{i=1}^{n} \log \left(u_{2 i}\right) \\
& +\alpha_{1} \sum_{i=1}^{n} \log \left(1-u_{i 1}\right)+\alpha_{2} \sum_{i=1}^{n} \log \left(1-u_{i 2}\right)-(1+\lambda) \sum_{i=1}^{n} \log \left(q_{i 1} q_{i 2}\right) \\
& -2 \sum_{i=1}^{n} \log \left[\left(1-u_{i 1}\right)^{\alpha_{1}}+u_{i 1}^{\alpha_{1}}\right]-2 \sum_{i=1}^{n} \log \left[\left(1-u_{2 i}\right)^{\alpha_{2}}+u_{2 i}^{\alpha_{2}}\right] \\
& -\left(2+\frac{1}{\lambda}\right) \sum_{i=1}^{n} \log \left[q_{1 i}^{\lambda}+q_{2 i}^{\lambda}-1\right],
\end{aligned}
$$

where

$$
q_{i k}=\frac{\left(1-u_{i k}\right)^{\alpha_{k}}}{\left(1-u_{i k}\right)^{\alpha_{k}}+u_{i k}^{\alpha_{k}}}, \quad u_{i k}=\exp \left[-\exp \left(z_{i k}\right)\right], \quad z_{i k}=\frac{y_{i k}-\boldsymbol{x}_{i k}^{T} \boldsymbol{\beta}_{k}}{\sigma_{k}}, \quad i=1, \ldots, n, k=1,2 .
$$

The MLEs of the parameters in $\psi$ can be obtained by maximizing the functions (3.2) and (3.3). The MLEs of the regression coefficients and unknown parameters are the solutions of the nonlinear equations of the score vectors $U_{\lambda}=0, U_{\boldsymbol{\theta}_{1}}=\mathbf{0}$ and $U_{\boldsymbol{\theta}_{2}}=\mathbf{0}$. We adopt iterative methods to determine the estimates. The maxBFGS routine in the matrix programming Ox language (Doornik, 2007) has been used for maximizing the $\log$-likelihood function $\ell(\boldsymbol{\psi})$. Initial values for $\boldsymbol{\beta}_{k}, \sigma_{k}$ and $\lambda$ are taken from the fit of the Frank-LW and Clayton-LW bivariate regression models with $\alpha_{1}=\alpha_{2}=1$.

For interval estimation and hypothesis tests on the model parameters, we require the $(2 p+5) \times$ $(2 p+5)$ observed $(\ddot{\boldsymbol{L}}(\boldsymbol{\psi}))$ and expected $(\boldsymbol{I}(\boldsymbol{\psi}))$ information matrices evaluated numerically. Under general regularity conditions, we can construct approximate confidence intervals for the parameters based on the multivariate normal $N_{2 p+5}\left(0, \boldsymbol{I}(\widehat{\psi})^{-1}\right)$ distribution, where $\widehat{\psi}$ is the MLE of $\boldsymbol{\psi}$.

We can evaluate the maximum values of the unrestricted and restricted log-likelihoods to obtain likelihood ratio (LR) statistics for testing some sub-models of the Frank-LOLLW and ClaytonLOLLW models. For example, the test of $H_{0}: \alpha_{1}=\alpha_{2}=1$ versus $H: H_{0}$ is not true is equivalent to compare the Frank-LOLLW (or Clayton-LOLLW) and Frank-LW (or Clayton-LW) and the LR statistic reduces to

$$
w=2\left[l\left(\hat{\lambda}, \hat{\boldsymbol{\beta}}_{1}, \hat{\boldsymbol{\beta}}_{2}, \hat{\sigma}_{1}, \hat{\sigma}_{2}, \hat{\alpha}_{1}, \hat{\alpha}_{2}\right)-l\left(\tilde{\lambda}, \tilde{\boldsymbol{\beta}}_{1}, \tilde{\boldsymbol{\beta}}_{2}, \tilde{\sigma}_{1}, \tilde{\sigma}_{2}, 1,1\right)\right],
$$

where $\hat{\lambda}, \hat{\boldsymbol{\beta}}_{1}, \hat{\boldsymbol{\beta}}_{2}, \hat{\sigma}_{1}, \hat{\sigma}_{2}, \hat{\alpha}_{1}$, and $\hat{\alpha}_{2}$ are the MLEs under $H$ and $\tilde{\lambda}, \tilde{\boldsymbol{\beta}}_{1}, \tilde{\boldsymbol{\beta}}_{2}, \tilde{\sigma}_{1}$, and $\tilde{\sigma}_{2}$ are the estimates under $H_{0}$.

\subsection{Simulation study}

We perform a Monte Carlo simulation study to assess the finite sample behavior of the MLEs of the parameters in $\psi$. The results are obtained from 1,000 Monte Carlo simulations done using the optim function in the R software. In each replication, a random sample of size $n$ is drawn from the FrankLOLLW regression model and the parameters are estimated by maximum likelihood. The samples denoted by $\left(y_{1 k}, x_{1 k}\right), \ldots,\left(y_{n k}, x_{n k}\right)$, for $k=1,2$, where the covariates $x_{i k}$ are generated from a uniform distribution in the range $(0,1)$. The artificial data are generated according to the following steps. First, 
Table 1: Mean estimates and MSEs of the MLEs of the parameters in the Frank-LOLLW regression model

\begin{tabular}{|c|c|c|c|c|c|c|c|c|}
\hline \multirow{2}{*}{ Parameter } & \multicolumn{2}{|c|}{$N=200$} & \multicolumn{2}{|c|}{$N=300$} & \multicolumn{2}{|c|}{$N=400$} & \multicolumn{2}{|c|}{$N=600$} \\
\hline & Mean & MSE & Mean & MSE & Mean & MSE & Mean & MSE \\
\hline$\alpha_{1}$ & 0.4951 & 0.0053 & 0.4956 & 0.0036 & 0.4968 & 0.0028 & 0.4975 & 0.0019 \\
\hline$\sigma_{1}$ & 0.9821 & 0.0140 & 0.9868 & 0.0097 & 0.9920 & 0.0071 & 0.9910 & 0.0048 \\
\hline$\beta_{01}$ & 4.0123 & 0.0390 & 4.0029 & 0.0261 & 4.0056 & 0.0174 & 4.0092 & 0.0119 \\
\hline$\beta_{11}$ & -1.0075 & 0.0925 & -0.9932 & 0.0571 & -1.0055 & 0.0430 & -1.0047 & 0.0275 \\
\hline$\alpha_{2}$ & 0.9935 & 0.0378 & 0.9939 & 0.0240 & 1.0015 & 0.0183 & 0.9984 & 0.0113 \\
\hline$\sigma_{2}$ & 0.4928 & 0.0066 & 0.4937 & 0.0043 & 0.4977 & 0.0033 & 0.4976 & 0.0021 \\
\hline$\beta_{02}$ & 3.4983 & 0.0049 & 3.5012 & 0.0032 & 3.5013 & 0.0023 & 3.4990 & 0.0016 \\
\hline$\beta_{12}$ & -0.4966 & 0.0135 & -0.5013 & 0.0089 & -0.4998 & 0.0059 & -0.4971 & 0.0045 \\
\hline$\lambda$ & 3.0281 & 0.2230 & 3.0245 & 0.1459 & 3.0276 & 0.1196 & 2.9977 & 0.0781 \\
\hline
\end{tabular}

MSE = mean square error; MLE = maximum likelihood estimate.

we generate $y_{i 1}=\mu_{1 i}+\sigma_{1} \log \left[-\log \left(1-A_{1 i}\right)\right]$, where $\mu_{1 i}=\beta_{01}+\beta_{11} x_{1 i}, A_{1 i}=u_{1 i}^{1 / \alpha_{1}} /\left[u_{1 i}^{1 / \alpha_{1}}+\left(1-u_{1 i}\right)^{1 / \alpha_{1}}\right]$ and $u_{i 1} \sim U(0,1)$.

Next, $y_{i 2}$ is generated using a random variable $w_{i} \sim U(0,1)$ to obtain $u_{2 i}=-(1 / \lambda) \log \left[1+w_{i}\left(\mathrm{e}^{-\lambda}-\right.\right.$ $\left.1) /\left(\mathrm{e}^{-\lambda u_{1 i}}-w_{i}\left(\mathrm{e}^{-\lambda u_{1 i}}-1\right)\right)\right]$, considering $y_{i 2}=\mu_{2 i}+\sigma_{2} \log \left[-\log \left(1-A_{2 i}\right)\right]$, where $\mu_{2 i}=\beta_{02}+\beta_{12} x_{2 i}$ and $A_{2 i}=u_{2 i}^{1 / \alpha_{2}} /\left[u_{2 i}^{1 / \alpha_{2}}+\left(1-u_{2 i}\right)^{1 / \alpha_{2}}\right]$.

The simulation study is performed for $n=200,300,400$, and 600. We consider the following values for the true parameters of the model $\alpha_{1}=0.5, \sigma_{1}=1.0, \beta_{01}=4.0, \beta_{11}=-1.0, \alpha_{2}=1.0$, $\sigma_{2}=0.5, \beta_{02}=3.5, \beta_{12}=-0.5$, and $\lambda=3.0$.

Table 1 displays the averages of the MLEs (mean) and the mean square errors (MSEs) given by $\operatorname{MSE}(\hat{\psi})=\operatorname{Var}(\hat{\psi})+[\operatorname{Bias}(\hat{\psi})]^{2}$. We can note that the estimates are closer to the true values and that the MSE values decrease when $n$ increases.

The results of the Monte Carlo in Table 1 indicate that the MSEs of the MLEs of the parameters decay toward zero when the sample size increases, as expected under standard asymptotic theory. As $n$ increases, the means of the estimates of the parameters tend to be closer to the true parameter values. This fact supports that the asymptotic normal distribution provides an adequate approximation to the finite sample distribution of the MLEs. The normal approximation can often be improved by using bias adjustments to these estimators.

\section{Influence diagnostics}

Performing a sensitivity analysis is strongly advisable since regression models are often sensitive to underlying model assumptions. Cook (1986) used this idea to motivate the assessment of the influence analysis. He also suggested that more confidence can be given to a model, which is relatively stable under small modifications. The best known perturbation schemes are based on local influence in which the effects are studied when completely removing cases from the analysis. This reasoning forms the basis for our local influence methodology and in doing so it will be possible to determine which subjects might be influential for the analysis.

Another approach is suggested by Cook (1986), where weights are given to observations instead of removing them. Local influence calculation can be carried out for model (3.2). If likelihood displacement $\operatorname{LD}(\omega)=2\left\{l(\hat{\psi})-l\left(\hat{\psi}_{\omega}\right)\right\}$ is used, where $\hat{\psi}_{\omega}$ denotes the MLE under the perturbed model, the normal curvature for $\psi$ at the direction $\boldsymbol{d},\|\boldsymbol{d}\|=1$, is given by $C_{\boldsymbol{d}}(\boldsymbol{\psi})=2\left|\boldsymbol{d}^{T} \boldsymbol{\Delta}^{T}[\ddot{\boldsymbol{L}}(\boldsymbol{\psi})]^{-1} \boldsymbol{\Delta d}\right|$. Here, $\Delta$ is a $(2 p+5) \times n$ matrix that depends on the perturbation scheme, whose elements are given by $\Delta_{v i}=\partial^{2} l(\psi \mid \omega) / \partial \psi_{\nu} \partial \omega_{i}(i=1,2, \ldots, n$ and $v=1,2, \ldots, 2 p+5)$ evaluated at $\hat{\psi}$ and $\omega_{0}$, where $\omega_{0}$ is the no perturbation vector (Cook, 1986). 
We can also determine normal curvatures $C_{\boldsymbol{d}}(\lambda), C_{\boldsymbol{d}}\left(\boldsymbol{\theta}_{1}\right)$, and $C_{\boldsymbol{d}}\left(\boldsymbol{\theta}_{2}\right)$ to perform various index plots, for instance, the index plot of $\boldsymbol{d}_{\max }$, the eigenvector corresponding to $C_{\boldsymbol{d}_{\max }}$, the largest eigenvalue of the matrix $\boldsymbol{B}=-\boldsymbol{\Delta}^{T}[\ddot{\boldsymbol{L}}(\boldsymbol{\psi})]^{-1} \boldsymbol{\Delta}$ and the index plots of $C_{\boldsymbol{d}_{i}}(\lambda), C_{\boldsymbol{d}_{i}}\left(\boldsymbol{\theta}_{1}\right)$, and $C_{\boldsymbol{d}_{i}}\left(\boldsymbol{\theta}_{2}\right)$, so-called total local influence (Lesaffre and Verbeke, 1998), where $\boldsymbol{d}_{i}$ denotes an $n \times 1$ vector of zeros with one at the $i^{t h}$ position. Thus, the curvature at direction $\boldsymbol{d}_{i}$ takes the form $C_{i}=2\left|\boldsymbol{\Delta}_{i}^{T}[\ddot{\boldsymbol{L}}(\psi)]^{-1} \boldsymbol{\Delta}_{i}\right|$, where $\boldsymbol{\Delta}_{i}^{T}$ denotes the $i^{t h}$ row of $\Delta$. It is usual to point out those cases such that $C_{i} \geq 2 \bar{C}$, where $\bar{C}=1 / n \sum_{i=1}^{n} C_{i}$.

Next, we evaluate, for three perturbation schemes, the matrix

$$
\Delta=\left(\Delta_{v i}\right)_{(2 p+5) \times n}=\left(\frac{\partial^{2} l(\psi \mid \omega)}{\partial \psi_{v} \partial \omega_{i}}\right)_{(2 p+5) \times n},
$$

where $v=1,2, \ldots, 2 p+5$ and $i=1,2, \ldots, n$, considering the model defined in (3.1) and its loglikelihood function given by (3.2) and (3.3).

\subsubsection{Case-weight perturbation}

Consider the vector of weights $\omega=\left(\omega_{1}, \ldots, \omega_{n}\right)^{T}$. In this case, the perturbed log-likelihood function takes the form:

- Frank-LOLLW model

$$
\begin{aligned}
l(\psi \mid \omega)= & \sum_{i=1}^{n} \omega_{i}\left(z_{i 1}+z_{i 2}\right)+\alpha_{1} \sum_{i=1}^{n} \omega_{i} \log \left(u_{i 1}\right)+\alpha_{2} \sum_{i=1}^{n} \omega_{i} \log \left(u_{i 2}\right) \\
& +\lambda \sum_{i=1}^{n} \omega_{i}\left(1+q_{i 1}+q_{i 2}\right)+\left(\alpha_{1}-1\right) \sum_{i=1}^{n} \omega_{i} \log \left(1-u_{i 1}\right)+\left(\alpha_{2}-1\right) \sum_{i=1}^{n} \omega_{i} \log \left(1-u_{i 2}\right) \\
& -2 \sum_{i=1}^{n} \omega_{i} \log \left\{\exp (\lambda)-\exp \left[\lambda\left(1+q_{i 1}\right)\right]+\exp \left[\lambda\left(q_{i 1}+q_{i 2}\right)\right]-\exp \left[\lambda\left(1+q_{i 2}\right)\right]\right\} \\
& -2 \sum_{i=1}^{n} \omega_{i} \log \left[\left(1-u_{i 1}\right)^{\alpha_{1}}+u_{i 1}^{\alpha_{1}}\right]-2 \sum_{i=1}^{n} \omega_{i} \log \left[\left(1-u_{i 2}\right)^{\alpha_{2}}+u_{i 2}^{\alpha_{2}}\right]
\end{aligned}
$$

- Clayton-LOLLW

$$
\begin{aligned}
l(\boldsymbol{\psi} \mid \omega)= & \sum_{i=1}^{n} \omega_{i}\left(z_{i 1}+z_{i 2}\right)+\alpha_{1} \sum_{i=1}^{n} \omega_{i} \log \left(u_{i 1}\right)+\alpha_{2} \sum_{i=1}^{n} \omega_{i} \log \left(u_{2 i}\right) \\
& +\alpha_{1} \sum_{i=1}^{n} \omega_{i} \log \left(1-u_{i 1}\right)+\alpha_{2} \sum_{i=1}^{n} \omega_{i} \log \left(1-u_{i 2}\right)-(1+\lambda) \sum_{i=1}^{n} \omega_{i} \log \left(q_{i 1} q_{i 2}\right) \\
& -2 \sum_{i=1}^{n} \omega_{i} \log \left[\left(1-u_{i 1}\right)^{\alpha_{1}}+u_{i 1}^{\alpha_{1}}\right]-2 \sum_{i=1}^{n} \omega_{i} \log \left[\left(1-u_{2 i}\right)^{\alpha_{2}}+u_{2 i}^{\alpha_{2}}\right] \\
& -\left(2+\frac{1}{\lambda}\right) \sum_{i=1}^{n} \omega_{i} \log \left[q_{1 i}^{\lambda}+q_{2 i}^{\lambda}-1\right]
\end{aligned}
$$

where $z_{i k}=y_{i k}-\boldsymbol{x}_{i k}^{T} \boldsymbol{\beta}_{k} / \sigma_{k}, 0 \leq \omega_{i} \leq 1, \omega_{0}=(1, \ldots, 1)^{T}, i=1, \ldots, n$ and $k=1,2$. Note that $q_{i 1}, q_{i 2}$, $u_{i 1}$ and $u_{i 2}$ are defined in equations (3.2) and (3.3). The matrix elements of $\boldsymbol{\Delta}=\left(\boldsymbol{\Delta}_{\lambda}, \boldsymbol{\Delta}_{\boldsymbol{\theta}_{1}}, \boldsymbol{\Delta}_{\boldsymbol{\theta}_{2}}\right)^{T}$ can be evaluated numerically. 


\subsubsection{Response perturbation}

Consider that each $y_{i 1}$ and $y_{i 2}$ is perturbed as $y_{i 1 w}=y_{i 1}+\omega_{i} S_{y 1}$ and $y_{i 2 w}=y_{i 2}+\omega_{i} S_{y 2}$, respectively, where $S_{y k}$ is a scale factor that may be estimated by the standard deviation of $Y_{k}$ and $\omega_{i} \in \mathbb{R}$.

Here, the perturbed log-likelihood function can be expressed as

- Frank-LOLLW model

$$
\begin{aligned}
l(\boldsymbol{\psi} \mid \boldsymbol{\omega})= & n \log \left\{\frac{\lambda \alpha_{1} \alpha_{2}[\exp (\lambda)-1]}{\sigma_{1} \sigma_{2}}\right\}+\sum_{i=1}^{n}\left(z_{i 1}^{*}+z_{i 2}^{*}\right)+\alpha_{1} \sum_{i=1}^{n} \log \left(u_{i 1}^{*}\right)+\alpha_{2} \sum_{i=1}^{n} \log \left(u_{i 2}^{*}\right) \\
& +\lambda \sum_{i=1}^{n}\left(1+q_{i 1}^{*}+q_{i 2}^{*}\right)+\left(\alpha_{1}-1\right) \sum_{i=1}^{n} \log \left(1-u_{i 1}^{*}\right)+\left(\alpha_{2}-1\right) \sum_{i=1}^{n} \log \left(1-u_{i 2}^{*}\right) \\
& -2 \sum_{i=1}^{n} \log \left\{\exp (\lambda)-\exp \left[\lambda\left(1+q_{i 1}^{*}\right)\right]+\exp \left[\lambda\left(q_{i 1}^{*}+q_{i 2}^{*}\right)\right]-\exp \left[\lambda\left(1+q_{i 2}^{*}\right)\right]\right\} \\
& -2 \sum_{i=1}^{n} \log \left[\left(1-u_{i 1}^{*}\right)^{\alpha_{1}}+u_{i 1}^{* \alpha_{1}}\right]-2 \sum_{i=1}^{n} \log \left[\left(1-u_{i 2}\right)^{* \alpha_{2}}+u_{i 2}^{* \alpha_{2}}\right]
\end{aligned}
$$

- Clayton-LOLLW model

$$
\begin{aligned}
l(\boldsymbol{\psi} \mid \boldsymbol{\omega})= & n \log \left[\frac{(1+\lambda) \alpha_{1} \alpha_{2}}{\sigma_{1} \sigma_{2}}\right]+\sum_{i=1}^{n}\left(z_{i 1}^{*}+z_{i 2}^{*}\right)+\alpha_{1} \sum_{i=1}^{n} \log \left(u_{i 1}^{*}\right)+\alpha_{2} \sum_{i=1}^{n} \log \left(u_{2 i}^{*}\right) \\
& +\alpha_{1} \sum_{i=1}^{n} \log \left(1-u_{i 1}^{*}\right)+\alpha_{2} \sum_{i=1}^{n} \log \left(1-u_{i 2}^{*}\right)-(1+\lambda) \sum_{i=1}^{n} \log \left(q_{i 1}^{*} q_{i 2}^{*}\right) \\
& -2 \sum_{i=1}^{n} \log \left[\left(1-u_{i 1}^{*}\right)^{\alpha_{1}}+u_{i 1}^{* \alpha_{1}}\right]-2 \sum_{i=1}^{n} \log \left[\left(1-u_{2 i}^{*}\right)^{\alpha_{2}}+u_{2 i}^{* \alpha_{2}}\right] \\
& -\left(2+\frac{1}{\lambda}\right) \sum_{i=1}^{n} \log \left[q_{1 i}^{* \lambda}+q_{2 i}^{* \lambda}-1\right],
\end{aligned}
$$

where

$q_{i k}^{*}=\frac{\left(1-u_{i k}^{*}\right)^{\alpha_{k}}}{\left(1-u_{i k}^{*}\right)^{\alpha_{k}}+u_{i k}^{* \alpha_{k}}}, \quad u_{i k}^{*}=\exp \left[-\exp \left(z_{i k}^{*}\right)\right], \quad z_{i 1}^{*}=\frac{y_{i 1}^{*}-\boldsymbol{x}_{i}^{T} \boldsymbol{\beta}_{1}}{\sigma_{1}}, \quad z_{i 2}^{*}=\frac{y_{i 2}^{*}-\boldsymbol{x}_{i}^{T} \boldsymbol{\beta}_{2}}{\sigma_{2}}$, $y_{i 1}^{*}=y_{i 1}+\omega_{i} S_{y 1}, \quad y_{i 2}^{*}=y_{i 2}+\omega_{i} S_{y 2}, \quad i=1, \ldots, n$ and $k=1,2$.

Again, the matrix elements of $\Delta=\left(\Delta_{\boldsymbol{\lambda}}, \Delta_{\boldsymbol{\theta}_{1}}, \boldsymbol{\Delta}_{\boldsymbol{\theta}_{2}}\right)^{T}$ can be obtained numerically.

\subsubsection{Explanatory variable perturbation}

Consider now an additive perturbation on a particular continuous explanatory variable, say $X_{t}$, by setting $x_{i t \omega}=x_{i t}+\omega_{i} S_{x}$, where $S_{x}$ is a scaled factor, $\omega_{i} \in \mathbb{R}$. This perturbation scheme leads to the following expressions for the log-likelihood function: 
- Frank-LOLLW model

$$
\begin{aligned}
l(\boldsymbol{\psi} \mid \boldsymbol{\omega})= & n \log \left\{\frac{\lambda \alpha_{1} \alpha_{2}[\exp (\lambda)-1]}{\sigma_{1} \sigma_{2}}\right\}+\sum_{i=1}^{n}\left(z_{i 1}^{\dagger}+z_{i 2}^{\dagger}\right)+\alpha_{1} \sum_{i=1}^{n} \log \left(u_{i 1}^{\dagger}\right)+\alpha_{2} \sum_{i=1}^{n} \log \left(u_{i 2}^{\dagger}\right) \\
& +\lambda \sum_{i=1}^{n}\left(1+q_{i 1}^{\dagger}+q_{i 2}^{\dagger}\right)+\left(\alpha_{1}-1\right) \sum_{i=1}^{n} \log \left(1-u_{i 1}^{\dagger}\right)+\left(\alpha_{2}-1\right) \sum_{i=1}^{n} \log \left(1-u_{i 2}^{\dagger}\right) \\
& -2 \sum_{i=1}^{n} \log \left\{\exp (\lambda)-\exp \left[\lambda\left(1+q_{i 1}^{\dagger}\right)\right]+\exp \left[\lambda\left(q_{i 1}^{\dagger}+q_{i 2}^{\dagger}\right)\right]-\exp \left[\lambda\left(1+q_{i 2}^{\dagger}\right)\right]\right\} \\
& -2 \sum_{i=1}^{n} \log \left[\left(1-u_{i 1}^{\dagger}\right)^{\alpha_{1}}+u_{i 1}^{\dagger \alpha_{1}}\right]-2 \sum_{i=1}^{n} \log \left[\left(1-u_{i 2}\right)^{\dagger \alpha_{2}}+u_{i 2}^{\dagger \alpha_{2}}\right] ;
\end{aligned}
$$

- Clayton-LOLLW model

$$
\begin{aligned}
l(\boldsymbol{\psi} \mid \boldsymbol{\omega})= & n \log \left[\frac{(1+\lambda) \alpha_{1} \alpha_{2}}{\sigma_{1} \sigma_{2}}\right]+\sum_{i=1}^{n}\left(z_{i 1}^{\dagger}+z_{i 2}^{\dagger}\right)+\alpha_{1} \sum_{i=1}^{n} \log \left(u_{i 1}^{\dagger}\right)+\alpha_{2} \sum_{i=1}^{n} \log \left(u_{2 i}^{\dagger}\right) \\
& +\alpha_{1} \sum_{i=1}^{n} \log \left(1-u_{i 1}^{\dagger}\right)+\alpha_{2} \sum_{i=1}^{n} \log \left(1-u_{i 2}^{\dagger}\right)-(1+\lambda) \sum_{i=1}^{n} \log \left(q_{i 1}^{\dagger} q_{i 2}^{\dagger}\right) \\
& -2 \sum_{i=1}^{n} \log \left[\left(1-u_{i 1}^{\dagger}\right)^{\alpha_{1}}+u_{i 1}^{\dagger \alpha_{1}}\right]-2 \sum_{i=1}^{n} \log \left[\left(1-u_{2 i}^{\dagger}\right)^{\alpha_{2}}+u_{2 i}^{\dagger \alpha_{2}}\right] \\
& -\left(2+\frac{1}{\lambda}\right) \sum_{i=1}^{n} \log \left[q_{1 i}^{\dagger \lambda}+q_{2 i}^{\dagger \lambda}-1\right],
\end{aligned}
$$

where

$$
\begin{aligned}
q_{i k}^{\dagger} & =\frac{\left(1-u_{i k}^{\dagger}\right)^{\alpha_{k}}}{\left(1-u_{i k}^{\dagger}\right)^{\alpha_{k}}+u_{i k}^{\dagger \alpha_{k}}}, \quad u_{i k}^{\dagger}=\exp \left[-\exp \left(z_{i k}^{\dagger}\right)\right], \quad z_{i k}^{\dagger}=\frac{y_{i k}-\boldsymbol{x}_{i}^{\dagger \top} \boldsymbol{\beta}_{k}}{\sigma_{k}}, \\
\boldsymbol{x}_{i}^{\dagger \top} \boldsymbol{\beta}_{k} & =\beta_{0 k}+\beta_{1 k} x_{i 1}+\beta_{2 k} x_{i 2}+\cdots+\beta_{t k}\left(x_{i t}+\omega_{i} S_{x}\right)+\cdots+\beta_{p k} x_{i p}, \quad i=1, \ldots, n \text { and } k=1,2 .
\end{aligned}
$$

The matrix elements of $\boldsymbol{\Delta}=\left(\boldsymbol{\Delta}_{\boldsymbol{\lambda}}, \boldsymbol{\Delta}_{\boldsymbol{\theta}_{1}}, \boldsymbol{\Delta}_{\boldsymbol{\theta}_{2}}\right)^{T}$ can be evaluated numerically.

\section{Application: the oral health-related quality data}

In this section, we consider a dataset provided by the Department of Community Dentistry, Division of Health Education and Health Promotion, Piracicaba Dental School, University of CampinasUNICAMP. The present study has the objective of assessing changes in schoolchildren's oral healthrelated quality of life (OHRQoL) in a three year follow-up exam and evaluating the impact of caries incidence on the OHRQoL of adolescents. In 2009, a baseline sample of 515 adolescents, representative of the 12-year-old population in the city of Juiz de Fora, Minas Gerais (Brazil), was evaluated (de Paula et al., 2012). The final sample, reevaluated 3 years after the baseline exam, is composed of 291 adolescents, and represents a follow-up rate of 56.5\%. Of these, $150(51.5 \%)$ are female and 238 $(81.8 \%)$ study at public schools. 
The OHRQoL, as the name indicates, involves quality of life aspects related to the mouth. The response variable is the assessment of the OHRQoL of children. The data are obtained from a questionnaire called the Children Perceptions Questionnaire (CPQ) administered to children between the ages of 11 and 14 years, say $\mathrm{CPQ}_{11-14}$. The same questionnaire is applied twice, first to establish the baseline (start of the study) and then three years later to the same children. Therefore, the responses are bivariate associated with each child. The $\mathrm{CPQ}_{11-14}$ determines the OHRQoL and is composed of 36 items, grouped into four health domains:

- Oral symptoms, composed of 6 questions;

- Functional limitations, composed of 9 questions;

- Emotional well-being, composed of 9 questions; and

- Social well-being, composed of 12 questions.

In each item there are questions about the frequency of events involving the teeth, lips and jaw in the past three months. Responses are given on a Likert scale from 1 to 4 : "none" = 0; "once or twice" $=1$; "sometimes" = 2; "often" = 3 ; and "very often" $=4$. This allows obtaining an overall score by adding the number of each item. Higher scores on the CPQ $11-14$ indicate worse OHRQoL, because individuals who say "often" or "very often", for example, will be more likely to have experienced toothaches or other dental problems. Another index used in the study is decayed, missing or filled teeth (DMFT), which reflects the caries experience in children's permanent teeth.

In this analysis, we consider the bivariate regression model by means of the Clayton and Frank copulas, and take the LOLLW distribution and its corresponding sub-models as marginal distributions. The variables employed are:

- $t_{i 1}=$ OHRQoL(0): Overall OHRQL score at baseline (measured at the start of the study);

- $t_{i 2}=$ OHRQoL(3): Overall OHRQL score at time of follow-up (measured three years later);

- $x_{i 1}$ : incidence of component $D$ of DMFT: If there is an increase in decayed teeth during the three years $(0=$ increased, 1 = no change, 2 = declined $)$;

- $x_{i 2}$ : incidence of component $M$ of DMFT: If there is an increase in missing teeth during the three years $(0=$ increased, 1 = no change $)$;

- $x_{i 3}$ : incidence of the $F$ component of DMFT: If there is an increase in the number of fillings/restorations during the three years $(0=$ smaller number of filled teeth - probably due to extraction, 1 $=$ no change, 2 = larger number of teeth with fillings);

- $x_{i 4}$ : total DMFT score: Sum of the scores of the three elements listed above - forming the complete number of permanent decayed, missing and filled permanent teeth. Therefore, in the case of the file, we dichotomize whether or not there is an increase in the DMFT index during the three years, subtracting the final - initial score $(0=$ increased, $1=$ no change $)$;

- $x_{i 5}$ : incidence of bleeding gums: The presence of bleeding gums is measured at the start and after the three years ( $0=$ no gum bleeding in 2009 and bleeding in 2012 [new cases], $1=$ gum bleeding continued, 2 = gum bleeding in 2009 and none in 2012 [cure]); 
Table 2: Descriptive statistics for the oral health-related quality dataset

\begin{tabular}{cccccccccc}
\hline \hline Data & Mean & Median & Mode & SD & Variance & Skewness & Kurtosis & Min & Max \\
\hline OHRQoL(0) & 25.471 & 18.0 & 4.0 & 23.421 & 548.547 & 1.224 & 0.872 & 0.10 & 106.0 \\
OHRQoL(3) & 20.896 & 15.0 & 0.1 & 19.712 & 388.588 & 1.340 & 1.431 & 0.10 & 90.0 \\
\hline \hline
\end{tabular}

- $x_{i 6}$ : need for dental treatment: This evaluated if there was any increasing in the need for dental treatment of the child during the three years, measured by the Dental Aesthetic Index. This index measures the social acceptability of the dental appearance through evaluation of 10 occlusal characteristics $(0=$ new cases/need for dental treatment in 2009 , but such need appeared during the three years, 1 = no change in characteristics, 2 = need for dental treatment in 2009 but not in 2012);

- $x_{i 7}$ : use of retainer or braces: This evaluated whether the child received orthodontic treatment (braces and/or retainer) at any time during the study period $(0=$ no, $1=$ yes $)$;

- $x_{i 8}$ : household crowding: This measured the change in the ratio of the number of people in the child's home to the number of bedrooms in 2009 and 2012 ( $0=$ increased, $1=$ remained unchanged, 2 = decreased);

- $x_{i 9}$ : general quality of life questionnaire: This refers to the difference in the OHRQoL between 2009 and 2012 ( 0 = worse, $1=$ remained unchanged, $2=$ improved $)$.

- $x_{i 10}$ : visit to dentist: This measured whether the child visited a dentist in the past three years, only asked in $2012(0=$ no, $1=$ yes $)$;

- $x_{i 11}$ : gender $(0=$ girl, 1 = boy $)$,

where $i=1, \ldots, 291$.

First, we present some descriptive statistics of the two-dimensional response variable. Table 2 gives a descriptive summary of these data showing different degrees of skewness and kurtosis. Figure 3(a) displays the plot of the bivariate distribution. The contour plots (with level curves) provide information about the normality and correlation at the same time. Figure 3(b) reveals a positive correlation between the variables OHRQoL(3) and OHRQoL(0), because the contour lines (level curves) are positioned around the main diagonal.

The Kendall tau rank correlation coefficient determined for the baseline and follow-up is $\tau=0.49$ ( $p$-value $<0.001$ ), indicating a positive association between the variables, as can also be noted in the dispersion plot in Figure 4(b). It is observed from the QQ plot. Figure 4(a) reveals that the data do not follow a bivariate normal distribution. In this paper, we use the copula method to assess the changes in the baseline and follow-up scores according to changes in the environmental and clinical profiles during the study period and assess the dependence between these scores. To explain the times until the occurrence or distinct event in a single individual, the bivariate regressions obtained through copulas are adopted. The Frank and Clayton's copulas given in Section 3 are used to model the data.

We analyze the dataset considering that the random variables $Y_{i 1}=\log \left(T_{i 1}\right)$ and $Y_{i 2}=\log \left(T_{i 2}\right)$ are related to the explanatory variables by a linear model

$$
\begin{aligned}
y_{i k}= & \beta_{0 k}+\beta_{11 k} D_{i 11}+\beta_{12 k} D_{i 12}+\beta_{2 k} x_{i 2}+\beta_{31 k} D_{i 31}+\beta_{32 k} D_{i 32}+\beta_{4 k} x_{i 4}+\beta_{51 k} D_{i 51}+\beta_{52 k} D_{i 52} \\
& +\beta_{61 k} D_{i 61}+\beta_{62 k} D_{i 62}+\beta_{7 k} x_{i 7}+\beta_{81 k} D_{i 81}+\beta_{82 k} D_{i 82}+\beta_{91 k} D_{i 91}+\beta_{92 k} D_{i 92}+\beta_{10 k} x_{i 10} \\
& +\beta_{1 k} x_{i 11}+\sigma_{k} z_{i k}, \quad i=1, \ldots, 291, k=1,2,
\end{aligned}
$$




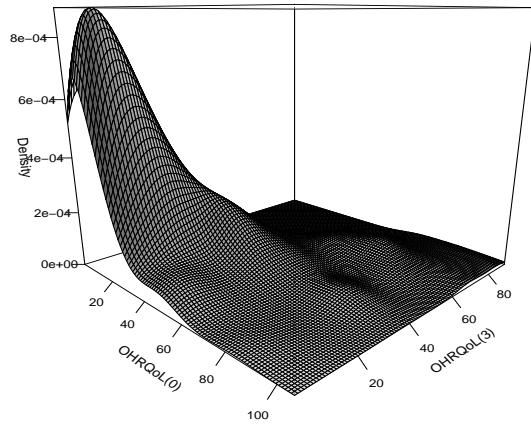

(a)

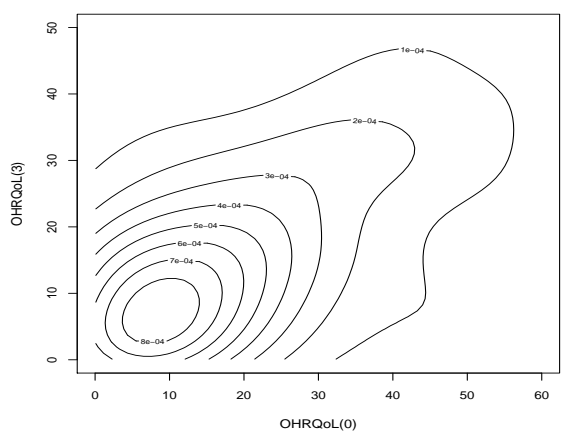

(b)

Figure 3: (a) Bivariate distribution; (b) Positive correlation between the variables $O H R Q O L(0)$ and $O H R Q o L(3)$.

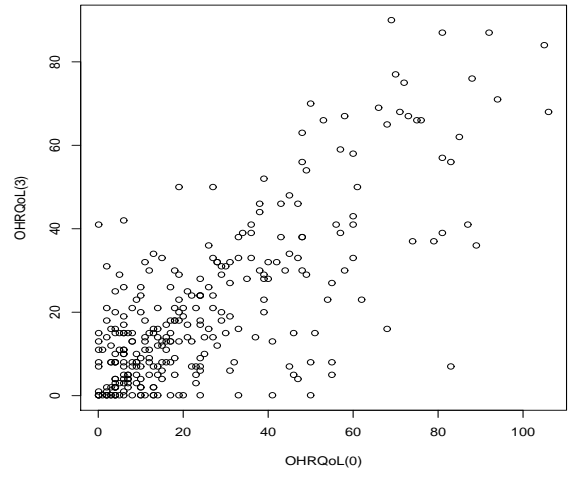

(a)

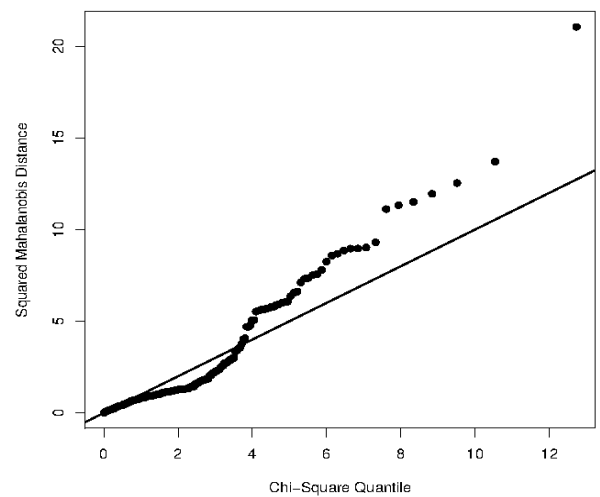

(b)

Figure 4: (a) The dispersion plot OHRQoL(3) versus OHRQoL(0) and (b) QQ plot.

where $z_{1}$ and $z_{2}$ are the random errors of the model with joint distribution given in equations (2.13) and (2.14). Note that the variables $\left(D_{11}, D_{12}\right),\left(D_{31}, D_{32}\right),\left(D_{51}, D_{52}\right),\left(D_{61}, D_{62}\right),\left(D_{81}, D_{82}\right),\left(D_{91}, D_{92}\right)$ are dummy variables corresponding to the variables $x_{1}, x_{3}, x_{5}, x_{6}, x_{8}$, and $x_{9}$, respectively.

We evaluate the MLEs of the model parameters using the NLMixed procedure in SAS. The values of the global deviance (GD), Akaike information criterion (AIC), consistent Akaike information criterion (CAIC), and Bayesian information criterion (BIC) statistics are listed in Tables 3 and 4. The lowest values of the information criteria correspond to the Frank-LOLLW bivariate regression model, which provides a better fit to the current data than the other models.

From the figures in Tables 3 and 4, we note that for the Clayton and Frank's copulas, the model that provides the best fit (lowest value of the AIC, CAIC, and BIC statistics), is the one which considers the LOLLW distribution in both components. Therefore, to decide which of the copulas to use in the subsequent analysis, we perform the generalized likelihood ratio test (GLRT) for discriminating among non-nested models as presented next (Cameron and Trivedi, 1998). The value of the GLRT statistic is 10.4664. Since this value is greater than 1.96, we reject the null hypothesis (at the 5\% 
Table 3: GD, AIC, BIC, and CAIC statistics for the Clayton-LOLLW bivariate regression model and submodels based on the oral health-related quality dataset

\begin{tabular}{|c|c|c|c|c|}
\hline $\begin{array}{l}\text { Bivariate regression models } \\
\qquad Y_{i 1} \times Y_{i 2}\end{array}$ & GD & AIC & CAIC & $\mathrm{BIC}$ \\
\hline LOLLW $\times$ LOLLW & $\mathbf{1 7 9 9 . 8 2 0 7}$ & 1881.8207 & 1895.6520 & 2032.4269 \\
\hline LOLLW × LOLLE & 1818.9485 & 1898.9485 & 1912.0685 & 2045.8814 \\
\hline LOLLW × LW & 1825.1329 & 1905.1329 & 1918.2529 & 2052.0658 \\
\hline LOLLW $\times$ LE & 1827.5811 & 1905.5811 & 1918.0114 & 2048.8407 \\
\hline LOLLE $\times$ LOLLW & 1811.3240 & 1891.3240 & 1904.4440 & 2038.2569 \\
\hline LW ×LOLLW & 1809.4347 & 1889.4347 & 1902.5547 & 2036.3677 \\
\hline LE $\times$ LOLLW & 1812.0360 & 1890.0360 & 1902.4663 & 2033.2956 \\
\hline $\mathrm{LW} \times \mathrm{LW}$ & 1831.4803 & 1909.4803 & 1921.9106 & 2052.7399 \\
\hline $\mathrm{LW} \times \mathrm{LE}$ & 1833.1898 & 1909.1898 & 1920.9517 & 2048.7761 \\
\hline $\mathrm{LE} \times \mathrm{LW}$ & 1837.7974 & 1913.7974 & 1925.5593 & 2053.3837 \\
\hline $\mathrm{LE} \times \mathrm{LE}$ & 1842.0951 & 1916.0951 & 1927.2097 & 2052.0081 \\
\hline
\end{tabular}

GD = global deviance; AIC = Akaike information criterion; CAIC = consistent Akaike information criterion; BIC = Bayesian information criterion; LOLLW = log-odd-log-logistic-Weibull; LOLLE = log-odd log-logistic exponential; LW $=\log$-Weibull; LE = log-exponential.

Table 4: GD, AIC, BIC, and CAIC statistics for the Frank-LOLLW bivariate regression model and submodels based on the oral health-related quality dataset

\begin{tabular}{ccccc}
\hline \hline Bivariate regression models & GD & AIC & CAIC & BIC \\
$Y_{i 1} \times Y_{i 2}$ & & $\mathbf{1 7 6 7 . 4 9 7 2}$ & $\mathbf{1 7 8 1 . 3 2 8 5}$ & $\mathbf{1 9 1 8 . 1 0 3 5}$ \\
\hline LOLLW $\times$ LOLLW & $\mathbf{1 6 8 5 . 4 9 7 2}$ & 1790.6342 & 1803.7542 & 1937.5671 \\
LOLLW $\times$ LOLLE & 1710.6342 & 1794.4440 & 1807.5640 & 1941.3769 \\
LOLLW $\times$ LW & 1714.4440 & 1792.9614 & 1805.3917 & 1936.2210 \\
LOLLW $\times$ LE & 1714.9614 & 1784.0638 & 1797.1838 & 1930.9967 \\
LOLLE $\times$ LOLLW & 1704.0638 & 1782.3472 & 1795.4672 & 1929.2801 \\
LW $\times$ LOLLW & 1702.3472 & 1782.2458 & 1794.6761 & 1925.5054 \\
LE $\times$ LOLLW & 1704.2458 & 1804.8332 & 1817.2635 & 1948.0928 \\
LW $\times$ LW & 1726.8332 & 1803.4266 & 1815.1885 & 1943.0129 \\
LW $\times$ LE & 1727.4266 & 1806.0146 & 1817.7765 & 1945.6009 \\
LE $\times$ LW & 1730.0146 & 1805.9501 & 1817.0647 & 1941.8631 \\
LE $\times$ LE & 1731.9501 & CAIC consi \\
\hline \hline
\end{tabular}

GD = global deviance; AIC = Akaike information criterion; CAIC = consistent Akaike information criterion; BIC = Bayesian information criterion; LOLLW = log-odd-log-logistic-Weibull; LOLLE = log-odd log-logistic exponential; LW $=\log$-Weibull; LE = log-exponential.

significance level) of equivalence between the models (attributing the LOLLW distribution to both components of the copula) and considering both the Frank and Clayton's copulas. So, the best model fitted to the data is the one for the Frank copula. MLEs of the parameters for the Frank-LOLLW bivariate regression model are listed in Table 5.

\section{Sensibility analysis}

We apply the local influence theory developed in Section 4, where case-weight perturbation is used, and obtain the value of the maximum curvature $C_{\boldsymbol{d}_{\max }}=2.8461$. Figure 5(a) displays the plots of the eigenvector corresponding to $\boldsymbol{d}_{\max }$, and reveals that the observations $\sharp 61$ and $\sharp 86$ are again distinct in relation to others.

The influence of perturbations on the observed survival times is now analyzed (response variable perturbation). The value of the maximum curvature is $C_{\boldsymbol{d}_{\max }}=9.3510$. Figure 5(b) plots $\boldsymbol{d}_{\max }$ versus the observation index, where we note that there is no discrepant point. 
Table 5: Maximum likelihood estimates for the Frank-LOLLW bivariate regression model based on the oral health-related quality dataset

\begin{tabular}{crcccrcc}
\hline \hline Parameter & Estimate & SE & $p$-value & Parameter & Estimate & SE & $p$-value \\
\hline$\lambda$ & 6.5401 & 0.5822 & & $\beta_{122}$ & 0.1608 & 0.2993 & 0.5911 \\
$\beta_{01}$ & 2.8875 & 0.4801 & $<0.0100^{* *}$ & $\beta_{22}$ & -0.1228 & 0.3021 & 0.6843 \\
$\beta_{111}$ & 0.0882 & 0.1589 & 0.5789 & $\beta_{312}$ & -0.2325 & 0.2155 & 0.2806 \\
$\beta_{121}$ & 0.1117 & 0.2790 & 0.6888 & $\beta_{322}$ & -0.2052 & 0.3214 & 0.5233 \\
$\beta_{21}$ & 0.2284 & 0.2791 & 0.4133 & $\beta_{42}$ & -0.1673 & 0.1836 & 0.3622 \\
$\beta_{311}$ & -0.1450 & 0.1904 & 0.4463 & $\beta_{512}$ & -0.1270 & 0.1466 & 0.3863 \\
$\beta_{321}$ & 0.0117 & 0.2783 & 0.9664 & $\beta_{522}$ & 0.3009 & 0.2392 & 0.2084 \\
$\beta_{41}$ & 0.1433 & 0.1652 & 0.3855 & $\beta_{612}$ & 0.1642 & 0.3793 & 0.6651 \\
$\beta_{511}$ & -0.2423 & 0.1288 & 0.0599 & $\beta_{622}$ & 0.4168 & 0.3630 & 0.2509 \\
$\beta_{521}$ & 0.4393 & 0.2053 & $0.0323^{* *}$ & $\beta_{72}$ & 0.1300 & 0.1716 & 0.4488 \\
$\beta_{611}$ & 0.1012 & 0.3484 & 0.7714 & $\beta_{812}$ & 0.0469 & 0.1457 & 0.7473 \\
$\beta_{621}$ & 0.4567 & 0.3381 & 0.1768 & $\beta_{822}$ & -0.0366 & 0.1681 & 0.8277 \\
$\beta_{71}$ & 0.1086 & 0.1485 & 0.4648 & $\beta_{912}$ & 0.0019 & 0.1442 & 0.9897 \\
$\beta_{811}$ & 0.1763 & 0.1249 & 0.1579 & $\beta_{922}$ & 0.2005 & 0.1013 & $0.0477^{* *}$ \\
$\beta_{821}$ & 0.4036 & 0.1468 & $0.0060^{* *}$ & $\beta_{102}$ & -0.3069 & 0.1289 & $0.0173^{* *}$ \\
$\beta_{911}$ & 0.0873 & 0.1271 & 0.4921 & $\beta_{12}$ & -0.0504 & 0.0965 & 0.6016 \\
$\beta_{921}$ & 0.2182 & 0.0917 & $0.0173^{* *}$ & $\sigma_{1}$ & 0.5178 & 0.0644 \\
$\beta_{101}$ & -0.3948 & 0.1078 & $0.0003^{* *}$ & $\sigma_{2}$ & 0.5452 & 0.0540 \\
$\beta_{11}$ & -0.0575 & 0.0844 & 0.4960 & $\alpha_{1}$ & 0.4779 & 0.0723 \\
$\beta_{02}$ & 3.4165 & 0.4980 & $<0.0100^{* *}$ & $\alpha_{2}$ & 0.4377 & 0.0557 \\
$\beta_{112}$ & -0.0502 & 0.1907 & 0.7924 & & & \\
\hline \hline
\end{tabular}

SE $=$ standard error. ${ }^{* *}$ Significant at a level of $5 \%$.

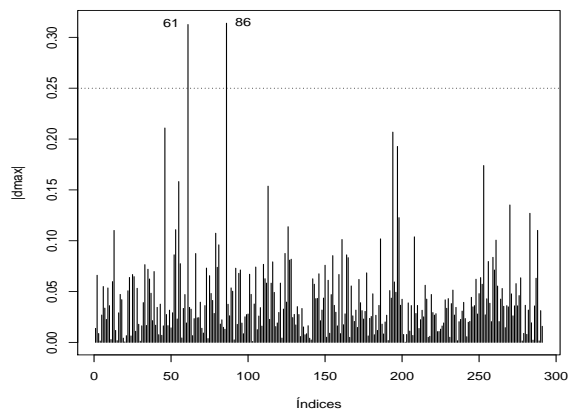

(a) Case-weight perturbation

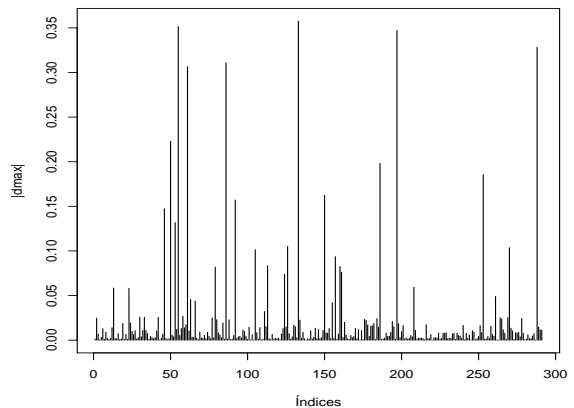

(b) Response perturbation

Figure 5: Index plot of $\boldsymbol{d}_{\max }$ for $\boldsymbol{\psi}$ on the oral health-related quality dataset.

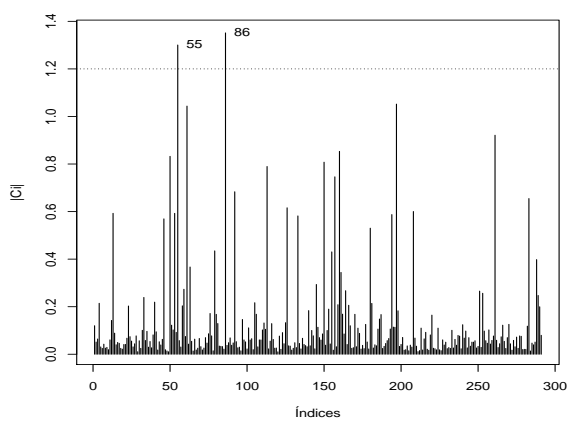

(a) Case-weight perturbation

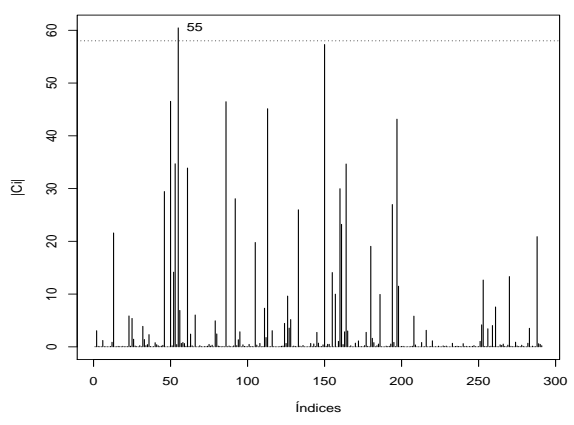

(b) Response perturbation

Figure 6: Total local influence for $\psi$ on the oral health-related quality dataset. 
Table 6: Maximum likelihood estimates for the Frank-LOLLW bivariate regression model removing observations $\sharp 55, \sharp 61$, and $\sharp 86$ based on the oral health-related quality dataset - final model

\begin{tabular}{lrlllrll}
\hline \hline Parameter & Estimate & SE & $p$-value & Parameter & Estimate & SE & $p$-value \\
\hline$\lambda$ & 6.2470 & 0.5690 & & $\beta_{122}$ & 0.2176 & 0.2930 & 0.4578 \\
$\beta_{01}$ & 2.8938 & 0.5000 & $<0.0100^{* *}$ & $\beta_{22}$ & 0.0184 & 0.2831 & 0.9480 \\
$\beta_{111}$ & 0.0447 & 0.1636 & 0.7846 & $\beta_{312}$ & -0.2316 & 0.2165 & 0.2847 \\
$\beta_{121}$ & 0.1323 & 0.2865 & 0.6442 & $\beta_{322}$ & -0.2537 & 0.3299 & 0.4418 \\
$\beta_{21}$ & 0.3324 & 0.2844 & 0.2424 & $\beta_{42}$ & -0.1568 & 0.1835 & 0.3928 \\
$\beta_{311}$ & -0.1455 & 0.1976 & 0.4615 & $\beta_{512}$ & -0.1673 & 0.1409 & 0.2351 \\
$\beta_{321}$ & -0.014 & 0.2893 & 0.9614 & $\beta_{522}$ & 0.1424 & 0.2510 & 0.5706 \\
$\beta_{41}$ & 0.1558 & 0.1689 & 0.3563 & $\beta_{612}$ & 0.0192 & 0.3662 & 0.9583 \\
$\beta_{511}$ & -0.2733 & 0.1314 & $0.0375^{* *}$ & $\beta_{622}$ & 0.3214 & 0.3516 & 0.3607 \\
$\beta_{521}$ & 0.3427 & 0.2268 & 0.1308 & $\beta_{72}$ & 0.1092 & 0.1680 & 0.5159 \\
$\beta_{611}$ & -0.0031 & 0.3617 & 0.9931 & $\beta_{812}$ & 0.0204 & 0.1432 & 0.8868 \\
$\beta_{621}$ & 0.4022 & 0.3546 & 0.2567 & $\beta_{822}$ & -0.0204 & 0.1619 & 0.8998 \\
$\beta_{71}$ & 0.0886 & 0.1482 & 0.5499 & $\beta_{912}$ & -0.0732 & 0.1433 \\
$\beta_{811}$ & 0.1540 & 0.1284 & 0.2304 & $\beta_{922}$ & 0.2876 & 0.1005 & 0.6095 \\
$\beta_{821}$ & 0.4016 & 0.1481 & $0.0067^{* *}$ & $\beta_{102}$ & -0.2534 & 0.1321 & $0.0542^{* *}$ \\
$\beta_{911}$ & 0.0255 & 0.1321 & 0.8472 & $\beta_{12}$ & -0.0935 & 0.1021 & 0.3596 \\
$\beta_{921}$ & 0.2838 & 0.0959 & $0.0031^{* *}$ & $\sigma_{1}$ & 0.5484 & 0.0692 \\
$\beta_{101}$ & -0.3470 & 0.1136 & $0.0023^{* *}$ & $\sigma_{2}$ & 0.5293 & 0.0522 \\
$\beta_{11}$ & -0.0988 & 0.0925 & 0.2852 & $\alpha_{1}$ & 0.5163 & 0.0803 \\
$\beta_{02}$ & 3.4341 & 0.4814 & $<0.0100^{* *}$ & $\alpha_{2}$ & 0.4248 & 0.0539 \\
$\beta_{112}$ & -0.1077 & 0.1886 & 0.5681 & & &
\end{tabular}

SE = standard error. ${ }^{* *}$ Significant at a level of $5 \%$.

The total local influence $C_{i}$ is shown in Figures 6 for case-weight and response perturbation, respectively. Observations $\sharp 55$ and $\sharp 86$ are distinct in relation to the others.

We conclude that the diagnostic analysis (global influence and local influence) detected the following three cases as potentially influential observations: $\sharp 55, \sharp 61$, and $\sharp 86$.

Observation $\sharp 55$ refers to the student with equal times OHRQoL(0) and OHRQoL(3), respectively, of 92 and 87, who: maintained the indices $C$ and $P$ of the CPOD, received fillings and did not increase the rate of caries, increased the CPOD index, had gum bleeding treated, maintained orthodontic treatment, did not use a retainer, maintained the same household crowding, suffered a deterioration in quality of life, had visited the dentist in the past 3 years and was female. The observation $\sharp 61$ refers to the student with equal times OHRQoL(0) and OHRQoL(3), respectively, to 66 and 69, who: maintained the indices $C, P$, and $O$ of the CPOD, maintained the CPOD index, continued to suffer gum bleeding, maintained orthodontic treatment, did not use a retainer, maintained the same household crowding, suffered deterioration in quality of life, had not visited the dentist for the past 3 years and was male. Finally, the observation $\sharp 86$ refers to the student with equal times OHRQoL(0) and OHRQoL(3), respectively, to 73 and 67, who: maintained the indices $C, P$, and $O$ of the CPOD, maintained the CPOD index, continued to have gum bleeding, maintained dental treatment, did not use a retainer, maintained the same household crowding, maintained the same quality of life, had not visited the dentist in the past 3 years and was male. Therefore, the MLEs of the fitted model after removing the observations $\sharp 55, \sharp 61$ and $\sharp 86$ are listed in Table 6 . This removal was also suggested by the researcher in charge of the study.

For example, the covariate $D_{82}$ (household crowding) is significant in the first half, meaning that there is a significant difference between the levels 0 (= increased) and 2 (= decreased). Figure 7 shows all the significant variables.

More information is provided by a visual comparison of the histogram of the data with the fitted 


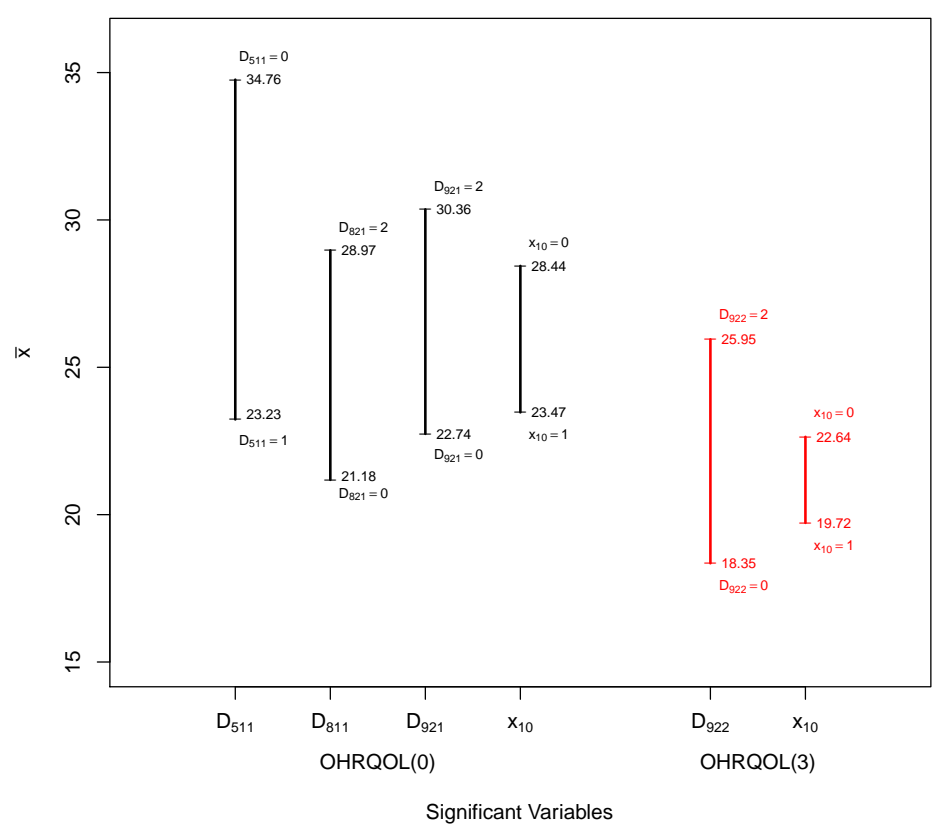

Figure 7: Plot of significant variables.

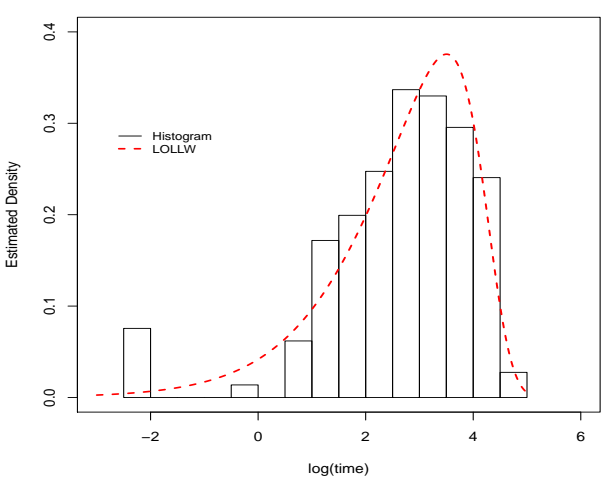

(a) OHRQoL(0)

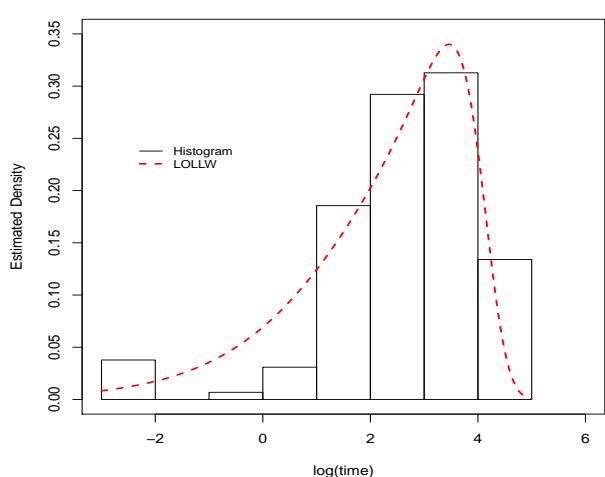

(b) OHRQoL(3)

Figure 8: Fitted LOLLW densities for the oral health-related quality dataset.

density functions displayed in Figure 8. We also conclude that the marginal models with LOLLW distribution provide an adequate fit to the data; therefore, the Frank-LOLLW bivariate regression model is more appropriate to explain these data.

\section{Conclusions}

We consider a parametric approach for bivariate survival data using location-scale marginal models having LOLLW distributions. The dependence structure between events is modeled using Archi- 
medean Frank and Clayton copulas. We present a general model that contain various sub-models. Our proposed bivariate model can help discriminate among models. We define the models, estimation techniques and diagnosis methods of global and total influence. These techniques were shown as important tools to evaluate the robustness of the bivariate response regression models. In the simulated examples, the local influence method is precise to detect the perturbed observations. In the example with the oral health-related quality dataset, the use of the proposed model and some sensitivity analysis methodology is illustrated. In the application section, we prove empirically that our bivariate model is more suitable to explain the quality of the oral health-related data.

\section{Acknowledgments}

We gratefully acknowledge financial support from CAPES and CNPq, Brazil.

\section{References}

Barriga GDC, Louzada-Neto F, Ortega EMM, and Cancho VG (2010). A bivariate regression model for matched paired survival data: local influence and residual analysis, Statistical Methods and Applications, 19, 477-495.

Chatterjee N and Shih J (2001). A bivariate cure-mixture approach for modeling familial association in diseases, Biometrics, 57, 779-786.

Cook RD (1986). Assessment of local influence, Journal of the Royal Statistical Society Series B (Methodological), 48, 133-169.

Cordeiro GM, Alizadeh M, Pescim RR, and Ortega EMM (2017a). The odd log-logistic generalized half-normal lifetime distribution: properties and applications, Communications in Statistics Theory and Methods, 46, 4195-4214.

Cordeiro GM, Alizadeh M, Ramires TG, and Ortega EMM (2017b). The generalized odd half-Cauchy family of distributions: properties and applications, Communications in Statistics - Theory and Methods, 46, 5685-5705.

Cameron AC and Trivedi PK (1998). Regression Analysis of Count Data, Cambridge University Press, New York.

Clayton DG (1978). A model for association in bivariate life tables and its application in epidemiological studies of familial tendency in chronic disease incidence, Biometrika, 65, 141-151.

Da Cruz JN, Ortega EMM, and Cordeiro GM (2016). The log-odd log-logistic Weibull regression model: modelling, estimation, influence diagnostics and residual analysis, Journal of Statistical Computation and Simulation, 86, 1516-1538.

Da Silva Braga A, Cordeiro GM, Ortega EMM, and da Cruz JN (2016). The odd log-logistic normal distribution: theory and applications in analysis of experiments, Journal of Statistical Theory and Practice, 10, 311-335.

De Paula JS, Leite IC, Almeida AB, Ambrosano GM, Pereira AC, and Mialhe FL (2012). The influence of oral health conditions, socioeconomic status and home environment factors on schoolchildren's self-perception of quality of life. Health Qual Life Outcomes, 10, 6.

Doornik JA (2007). An Object-Oriented Matrix Language: Ox 5, Timberlake Consultants Press, London.

Escobar LA and Meeker Jr WQ (1992). Assessing influence in regression analysis with censored data, Biometrics, 48, 507-528.

Eugene N, Lee C, and Famoye F (2002). Beta-normal distribution and its applications, Communications in Statistics - Theory and Methods, 31, 497-512. 
Fachini JB, Ortega EMM, and Cordeiro GM (2014). A bivariate regression model with cure fraction, Journal of Statistical Computation and Simulation, 84, 1580-1595.

Frank MJ (1979). On the simultaneous associativity of $F(x, y)$ and $x+y-F(x, y)$, Aequationes Mathematicae, 19, 194-226.

Genest C (1987). Frank's family of bivariate distributions, Biometrika, 74, 549-555.

Hashimoto EM, Ortega EMM, Cancho VG, and Cordeiro GM (2013). On estimation and diagnostics analysis in log-generalized gamma regression model for interval-censored data, Statistics, 47, 379-398.

Hashimoto EM, Ortega EMM, Cordeiro GM, and Cancho VG (2015). A new long-term survival model with interval-censored data, Sankhya B, 77, 207-239.

$\mathrm{He} \mathrm{W}$ and Lawless JF (2005). Bivariate location-scale models for regression analysis, with applications to lifetime data, Journal of the Royal Statistical Society Series B (Statistical Methodological), 67, 63-78.

Lesaffre E and Verbeke G (1998). Local influence in linear mixed models, Biometrics, 54, 570-582.

Nelsen RB (2006). An Introduction to Copulas (2nd ed), Springer, New York.

Núñez JSR (2005). Modelagem Bayesiana para Dados de Sobrevivência Bivariados Através de Cópulas (Doctoral dissertation), University of São Paulo, Brasil (in Portuguese).

Ortega EMM, Cordeiro GM, and Kattan MW (2013). The log-beta Weibull regression model with application to predict recurrence of prostate cancer, Statistical Papers, 54, 113-132.

Ortega EMM, Cordeiro GM, Campelo AK, Kattan MW, and Cancho VG (2015). A power series beta Weibull regression model for predicting breast carcinoma, Statistics in Medicine, 34, 1366-1388.

Ortega EMM, Cordeiro GM, Hashimoto EM, and Suzuki AK (2017). Regression models generated by gamma random variables with long-term survivors, Communications for Statistical Applications and Methods, 24, 43-65.

Ortega EMM, Lemonte AJ, Cordeiro GM, and da Cruz JN (2016). The odd Birnbaum-Saunders regression model with applications to lifetime data, Journal of Statistical Theory and Practice, 10, 780-804.

Pettitt AN and Bin Daud I (1989). Case-weight measures of influence for proportional hazards regression, Journal of the Royal Statistical Society. Series C (Applied Statistics), 38, 51-67.

Silva GO, Ortega EMM, and Cancho VG (2010). Log-Weibull extended regression model: estimation, sensitivity and residual analysis. Statistical Methodology, 7, 614-631. 